\title{
Special Issue on Systems on the Verge of Stability
}

Received: 2 September 2015 / Accepted: 3 September 2015 / Published online: 24 September 2015

(C) Springer-Verlag Wien 2015

The physics of quantum weakly bound systems permeates with common concepts several subfields, from condensed matter to particle physics. Recognition of the generality of the concepts and the cross disciplinarity of the techniques employed to treat few-body systems in Nuclear, Atomic and Condensed Matter physics close to stability led to the idea of composing a Special Issue in Few-Body Systems, to provide in a single volume this diversity of physical problems in different fields treated by common techniques and concepts. The importance of composing such a Special Issue was understood during the 7th International and Interdisciplinary Workshop on the Dynamics of Critically Stable Quantum Few-Body Systems (Critical Stability 2014) held in Santos, Brazil, from October 12 to 17, 2014, organised by Lauro Tomio, Mahir S. Hussein and myself, on the occasion of the 70th birthday of Prof. Mahir S. Hussein, when we had the opportunity to discuss this demand with colleagues from different fields with a common interest in quantum systems close to stability.

Much of our understanding of the physics of three-body systems in three-dimensions with short range interactions and large scattering lengths close to stability is owed to V. Efimov. He found that three-identical bosons have an infinite number of bound states condensing at the continuum threshold when the dimer is bound at zero energy. The phenomenum discovered by him is closely related to the Thomas collapse of the trimer for a zero range force. Behind it there is a continuum scale invariance broken to a discrete one, with states having properties rescaled by a common geometrical factor. The extension to more particles is still under debate. These large states are universal, in the sense that they are independent of the short range potential. It is thus not a surprise that, after the experimental discovery of such states in cold atoms a decade ago, Efimov physics has become a major branch of study in many areas. These weakly bound states have properties dominated by the classically forbidden region, and are therefore largely model independent. Nowadays, the research on the subject has widened and become interdisciplinary and no longer constrained to three-bosons nor to three-dimensions.

The problems found by searching for characteristics that are model independent in "systems on the verge of stability" transit between between different fields in physics and extend to lower dimensions, different geometries, boundary constraints and to many-body systems. Recognizing the utility of the research of universal or model independent features of quantum systems close to stability is the key to cross fields and the motivation to transfer techniques, methods and experience among different subfield of physics, obtained by solving problems found in Mathematical, Nuclear, Atomic and Condensed Matter physics.

The state-of-the-art contributions are timely, and I expect that the papers in this topical issue covering Efimov physics, weakly bound halo nuclei structure and reactions, from few to many body problems, new methods for solving few-body problems, and few-body systems under constraints or lower dimensions, will prove to be useful. I appreciate and thank all the contributing authors for their efforts, by presenting new ideas, very interesting discussions or a summary of their results.

T. Frederico $(\varangle)$

Instituto Tecnológico de Aeronáutica, DCTA, São José dos Campos, SP 12.228-900, Brazil

E-mail: tobias@ita.br 